\title{
Análise de um motor-foguete bi-propelente aplicada ao ensino de termodinâmica: um estudo de caso
}

Analysis of a bi-propellent rocket engine applied to teaching thermodynamics: a case study

\author{
Gustavo Farias Serra $\stackrel{10}{10}$, Maria Elisa Marciano Martinez ${ }^{20}$, Marcello Carvalho dos Reis ${ }^{30}$, Oswaldo

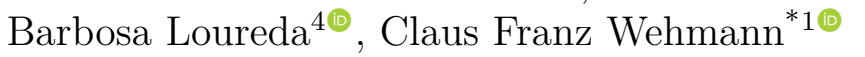 \\ ${ }^{1}$ Universidade Federal do Ceará, Fortaleza, CE, Brasil. \\ ${ }^{2}$ Instituto Nacional da Propriedade Industrial, São Paulo, SP, Brasil. \\ ${ }^{3}$ Meteora, Fortaleza, CE, Brasil \\ ${ }^{4}$ Acrux Aerospace Technologies, São José dos Campos, SP, Brasil
}

Recebido em 1 de maio de 2020. Revisado em 3 de julho de 2020. Aceito em 4 de julho de 2020.

\begin{abstract}
A importância do protagonismo do aluno no processo de aprendizagem é reconhecida há bastante tempo. Diversas metodologias têm sido desenvolvidas, como sala invertida e o ensino baseado em projeto, ou sua variante menos complexa o ensino baseado em estudo de caso. A termodinâmica e a mecânica dos fluidos são algumas das mais complexas disciplinas ensinadas na física e engenharias. Dentre suas principais dificuldades para os alunos, são a terminologia e os conceitos pouco conectados ao cotidiano, além da necessidade de em uma analise, haver a necessidade de conexão entre vários destes conceitos e uma matemática mais complexa que a encontrada na mecânica newtoniana. O estudo de caso é considerado por vários autores uma variante do ensino baseado em projetos mais simples de ser implementado e aceito pelos estudantes. Neste trabalho é realizado um estudo de caso sobre as relações termodinâmicas de um motor-foguete bi-propelente direcionada aos alunos de engenharia. A partir de um modelo simplificado do motor, uma modelagem matemática é feita utilizando a primeira lei da termodinâmica e suas implicações. Para a realização dos cálculos matemáticos a partir da análise, é considerado um motor cujo propelente e combustível são oxigênio líquido e hidrogênio líquido (LOX/LH2), respectivamente. Este estudo de caso está em sintonia com as diversas notícias sobre exploração espacial dos projetos da Índia, China ou do chamado new space.

Palavras-chave: Estudo de Caso, Termodinâmica, Aeroespacial, Foguetes.
\end{abstract}

The importance of the student's role in the learning process has been recognized for a long time. Several methodologies have been developed, such as inverted class and project-based teaching, or its less complex variant, case study-based teaching. Thermodynamics and fluid mechanics are some of the most complex disciplines taught in physics and engineering. Among its main difficulties for students, are the terminology and concepts little connected to everyday life, in addition to the need for an analysis, there is a need for connection between several of these concepts and a more complex mathematics than that found in Newtonian mechanics. The case study is considered by several authors to be a variant of teaching based on projects that are simpler to be implemented and accepted by students. In this work, a case study is carried out on the thermodynamic relationships of a bi-propellant rocket engine aimed at engineering students. From a simplified model of the engine, a mathematical modeling is done using the first law of thermodynamics and its implications. To perform the mathematical calculations from the analysis, an engine is considered whose propellant and fuel are liquid oxygen and liquid hydrogen (LOX / LH2), respectively. This case study is in line with The setting of the case can be made considering the various news about space exploration of projects in India, China or the so-called new space.

Keywords: Case Study, Thermodynamics, Aerospace, Rockets.

\section{Introdução}

Dentre as disciplinas que o aluno dos cursos de ciências e engenharias apresenta maiores dificuldades, sendo isso uma questão mundial, figuram a termodinâmica e a mecânica dos fluidos [1]. Segundo Dempster, Lee e Boyle [2], a dificuldade mais evidente está relacionada a fato destas disciplinas serem ricas em conceitos e vocabulá-

*Endereço de correspondência: claus.wehmann@ufc.br rios específicos e muitas vezes confuso. Normalmente é necessário a aplicação de diversos conceitos relacionados e equacionamento complexo para analisar qualquer problema resultando em confusão de como estas leis e equações interagem.

Desde a década de 1990, é reconhecido a importância do chamado Hands-on no ensino de engenharia [3] sendo adotada em diversos currículos, inclusive engenharia aeronáutica [4]. O protagonismo dos estudantes passou a 
ser reconhecido como fator importante na chamada construção do conhecimento. Diversas metodologias ativas vieram a ser formuladas, como a sala de aula invertida [5] e o ensino baseado em projeto (Project Based Learning $P B L)$ [6]. Esta última sendo uma das mais difundidas.

Apesar de sua difusão no meio acadêmico, o PBL, costuma ser criticado pela complexidade de sua implementação [7] e normalmente usado em situações que permeiam todo o curso. No caso de uma disciplina isolada, sugere-se o uso do estudo de caso [8]. O estudo de caso é uma variante simplificada, segundo Queiroz e Cabral [8], e esta simplicidade o faz mais compreensivo para os alunos, com mesmo benefício que o PBL [7].

A interatividade no ensino deve envoler, entre outras características, a interação física (hands-on), o emocional, intelectual e social segundo Bassoli [9], nessas categorias o aluno deve ser desafiado em seu conhecimento de tal forma que haja oportunidade de pensar e discutir, mas também seja motivado. Esta motivação pode ser obtida pela contextualização do desenvolvimento científico [10], através da interdisciplinaridade [11, 12, 13], e na situação de do estudo de caso, isso é alcançado quando o estudante aprende com casos próximos do real [14].

Neste trabalho, tanto a contextualização quanto o caso a ser estudado basea-se na problemática do desenvolvimento de um foguete, aproveitando o interesse recente sobre as atividades espaciais. O motor foguete é um motor térmico: converte o calor gerado pela queima dos propulsores - combustível e oxidante na câmara de combustão em energia de cinética dos gases de escape emergentes [15]. Há três tipos de foguetes com base em dois diferentes tipos de combustível: sólido, hibrido e líquido. Os primeiros foguetes de combustível sólido eram projéteis obtidos pela combustão de pólvora, usados como fogos de artifício ou armas pelos chineses já em 1232. O período áureo do desenvolvimento ocorreu principalmente durante a guerra fria, com o surgimento dos programas espaciais dos EUA e Rússia. O projeto de um motor- foguete é complexo e demorado, demandando incontáveis cálculos e simulações [16].

Atualmente o interesse no espaço, em foguetes, satélites e astronáutica em geral, está sendo renovado desde os impressionantes feitos do New Space como os espetaculares lançamentos da SpaceX, e o surgimento de das missões a lua da Índia, China e Israel [17, 18, 19]. Outra questão a ser aproveitada é a multidisciplinaridade inerente ao contexto aeroespacial [20].

Neste trabalho a Primeira Lei é explorada ao máximo, através de modelagens matemáticas que respeitem a conservação de energia.

Um esquema de um motor-foguete bi-propelente simplificado foi utilizado para a análise termodinâmica aqui desenvolvida, como segue na Figura 1 a seguir.

Os resultados desta análise podem ser apresentados na forma de uma aplicação prática da Primeira Lei da Termodinâmica.

\section{OBJETIVO}

Inicialmente deseja-se obter uma equação para a vazão total de combustível do tanque em função das propriedades termodinâmicas atingidas no motor e aplicá-las nos dados conhecidos de um foguete real. Além disso, espera-se rever e aplicar conhecimentos adquiridos anteriormente no curso de engenharia e buscar aprender mais a respeito da ciência de foguetes.

\section{METODOLOGIA}

Para relacionar as propriedades, utiliza-se principalmente a Primeira Lei da Termodinâmica. Ela permite encontrar uma relação entre as vazões de combustível e de oxidante. Seu enunciado diz que a taxa de variação de energia de um volume de controle é igual à taxa de transferência de energia por calor menos a taxa de transferência de energia por trabalho mais a taxa de energia que acompanha a

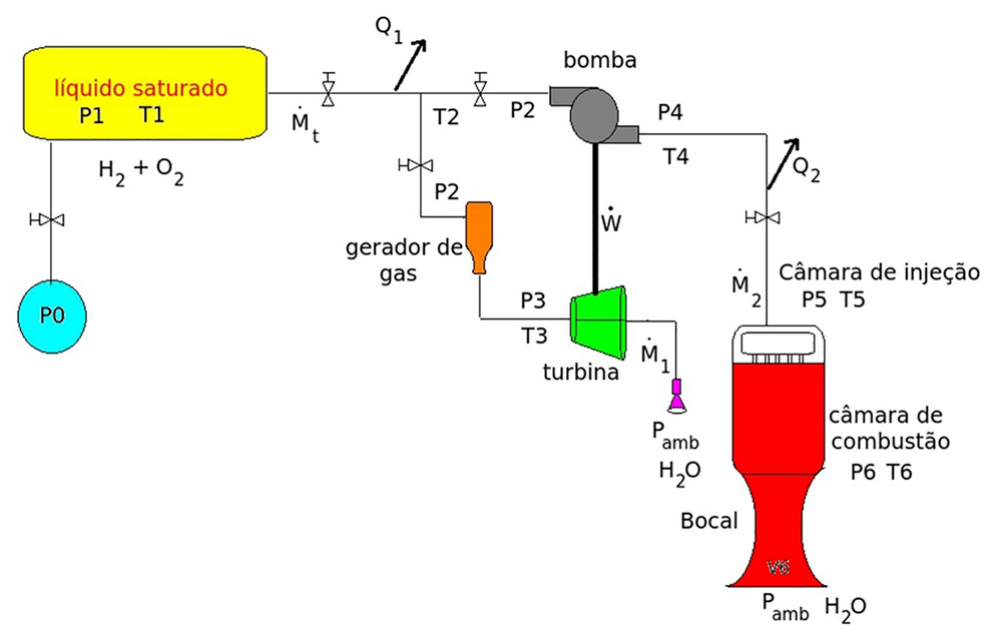

Figura 1: Esquema de um motor-foguete híbrido simplificado. Fonte: Autores. 
transferência de massa. Este enunciado está traduzido na seguinte equação [21]:

$$
\begin{gathered}
\Delta \mathrm{U}=\dot{Q}-\dot{W}+ \\
\dot{M}\left[h_{2}-h_{1}+\frac{V_{2}^{2}-V_{1}^{2}}{2}+g\left(z_{2}-z_{1}\right)\right]
\end{gathered}
$$

onde $\Delta \mathrm{U}$ - Variação total da energia, $\dot{Q}$ - Transferência de energia por calor, $\dot{W}$ - Transferência de energia por trabalho e o trecho entre colchetes se refere a Energia que acompanha a vazão mássica.

Considerando que o motor se trata de um regime permanente, pode-se afirmar que a variação da sua energia é nula.

Para fazer os cálculos, serão feitas algumas considerações a respeito do esquema do motor mostrado na introdução. Tais considerações podem ser modificadas para se adequarem as características de motores reais.

1. Hidrogênio e oxigênio serão líquidos incompressíveis;

2. Processos isentrópicos;

3. As reações são de queima completa;

4. Não há variação de energia potencial;

5. Temperatura e pressão na câmara de combustão $\left(\mathrm{P}_{6}\right.$ e $\mathrm{T}_{6}$ ): 48 bar e $3473 \mathrm{~K}$;

6. O gerador de gás tem pressão $\left(\mathrm{P}_{2}\right)$ de 1,25 vezes a pressão na entrada da turbina;

7. As eficiências da turbina e da bomba são iguais a 0,8 ou $80 \%$;

8. A razão das pressões da turbina $\left(\mathrm{P}_{3} / \mathrm{P}_{\mathrm{amb}}\right)$ é de 5 ;

9. As temperaturas $\mathrm{T}_{1}, \mathrm{~T}_{2}, \mathrm{~T}_{4}$ e $\mathrm{T}_{5}$ são as temperaturas de saturação para o combustível nas respectivas pressões.

\section{RESULTADOS}

O motor é dividido em quatro volumes de controle, que serão analisados separadamente.

Parte 1: tanques de combustível

Aqui o único ele-

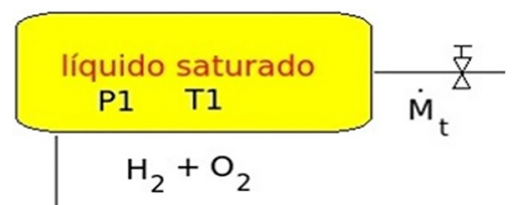
mento é a vazão de combustível + oxidante $\left(\dot{M}_{t}\right)$. Na imagem, os dois tanques são representados como um único tanque, mas o combustível e o oxidante se encontram apenas na câmara de combustão.

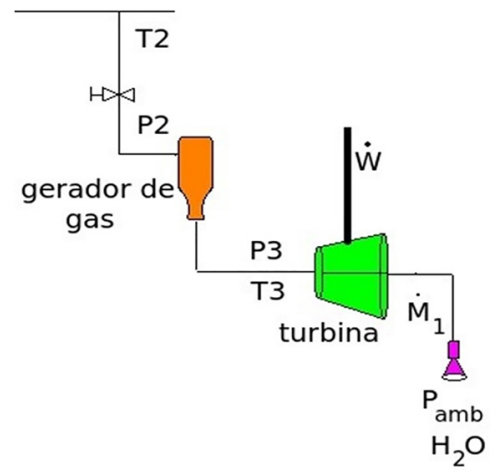

Parte 2: gerador de gás e turbina

Ao sair dos seus respectivos tanques, o combustível e o oxidante se dividem em duas partes: uma vai para o gerador de gás $\left(\dot{M}_{1}\right)$ e outra vai para a bomba $\left(\dot{M}_{2}\right)$.
No gerador de gás, a troca de energia é proveniente da reação entre hidrogênio e oxigênio, que produz uma variação na entalpia do volume de controle. Essa variação é contrabalanceada por uma variação na energia cinética.

$$
\dot{M}_{1}\left[\left(h_{2}-h_{3}\right)+\frac{V_{2}^{2}-V_{3}^{2}}{2}\right]=0
$$

Na turbina, tem-se a realização de trabalho pelo vapor de água se expandindo até a pressão ambiente. Desconsiderando a variação de energia cinética, esse trabalho realizado pode ser representado por:

$$
\dot{W}=\eta_{1} \dot{M}_{1}\left(h_{3}-h_{a m b}\right)
$$

Onde $\eta_{1}$ é a eficiência da turbina, igual a $80 \%$.

Parte 3: bomba

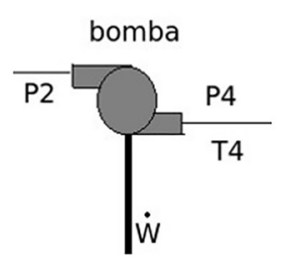

A bomba recebe o trabalho gerado pela turbina e o utiliza para aumentar a entalpia da mistura combustível + oxidante. A primeira Lei da Termodinâmica neste caso toma o seguinte formato:

$$
-\dot{W}=\dot{M}_{2} \eta_{2}\left(h_{4}-h_{2}\right)
$$

Onde $\eta_{2}$ é o rendimento da bomba, que é igual ao da turbina $(80 \%)$.

Parte 4: câmara de injeção

Na câmara de injeção, há uma queda de pressão de 95\%, ou seja: $P_{5}=0,95 \cdot P_{4}$

Na câmara de combustão, ocorre a reação exotérmica da segunda parte do combustível, transformando hidrogênio e oxigênio líquido em vapor de água.

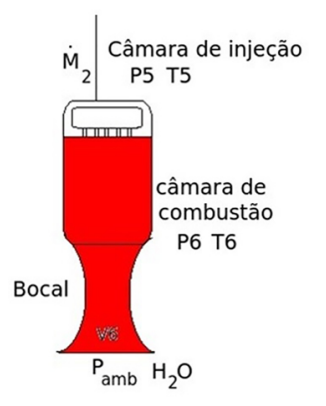

No bocal, o vapor de água está inicialmente estagnado, e, ao se expandir, atinge velocidades supersônicas transformando a sua entalpia em energia cinética. A velocidade de exaustão pode ser encontrada utilizando o impulso específico do foguete com a seguinte equação:

$$
v_{e}=g_{0} I_{s p}
$$

\section{Construção da fórmula}

As vazões $\dot{M}_{1}$ e $\dot{M}_{2}$ aparecem nas equações de transferência de energia da turbina e da bomba. É possível relacionar as duas, pois o mesmo trabalho produzido pela turbina é utilizado pela bomba. Daí tem-se (considerando que neste caso $\eta_{1}=\eta_{2}$ ):

$$
\begin{gathered}
\eta_{1} \dot{M}_{1}\left(h_{3}-h_{a m b}\right)=\dot{M}_{2} \eta_{2}\left(h_{4}-h_{2}\right) \\
\frac{\dot{M}_{1}}{\dot{M}_{2}}=\frac{\left(h_{2}-h_{4}\right)}{\left(h_{3}-h_{a m b}\right)}
\end{gathered}
$$


Substituindo na fórmula $\dot{M}_{t}=\dot{M}_{1}+\dot{M}_{2}$, encontra-se que:

$$
\begin{aligned}
& \dot{M}_{t}=\dot{M}_{2}\left[1+\frac{\left(h_{2}-h_{4}\right)}{\left(h_{3}-h_{a m b}\right)}\right] \\
& =\frac{W}{0,8 .\left(h_{2}-h_{4}\right)}\left[1+\frac{\left(h_{2}-h_{4}\right)}{\left(h_{3}-h_{a m b}\right)}\right]
\end{aligned}
$$

A potência pode ser dada como:

$$
\mathrm{W}=\rho g Q H=\dot{M}_{1} g H
$$

Onde $\rho$ é a densidade do líquido, Q é a vazão volumétrica, $\dot{M}_{1}=\rho Q$ e $\mathrm{H}$ é a altura de carga, que é determinada por:

$$
H=\frac{P_{4}-P_{2}}{\rho g}
$$

Após essas substituições, a nova equação da vazão será:

$$
\dot{M}_{t}=\frac{\dot{M}_{1}\left(P_{4}-P_{2}\right)}{0,8 . \rho\left(h_{2}-h_{4}\right)}\left[1+\frac{\left(h_{2}-h_{4}\right)}{\left(h_{3}-h_{a m b}\right)}\right]
$$

Onde $h_{3}$ e $h_{a m b}$ são referentes ao vapor de água e $\rho, \mathrm{h}_{2}$ e $\mathrm{h}_{4}$ são referentes à mistura de hidrogênio e oxigênio líquido. A entalpia resultante da mistura pode ser encontrada utilizando as frações de massa de cada componente.

$$
h=\% m_{L H 2} h_{L H 2}+\% m_{L O X} h_{L O X}
$$

A equação encontrada pode ser utilizada para aproximar um modelo da vazão de um foguete já existente que seja movido a LH2 e LOX, e pode ser útil para, se já se conhecer a vazão, encontrar as outras propriedades na trajetória do fluido. Ao analisar o caso experimentalmente com um foguete real, nota-se que a vazão de combustível e oxidante não é constante, e uma das vantagens dos foguetes híbrido e líquido é que esta vazão pode ser controlada. Porém é importante a construção desta fórmula, não apenas para criar um modelo matemático, mas também para incentivar os autores a compreender melhor o funcionamento de um motor de foguete, e explorar casos na literatura que abordem essa questão.

\section{CONCLUSÃO}

Neste trabalho foram analisadas as propriedades termodinâmicas de um motor foguete com bi propelente líquido, com o intuito didático para estudantes de engenharia demonstrando uma aplicação pratica da termodinâmica na engenharia aeroespacial.

O sistema de a propulsão foi dividido em quatro volumes de controle para facilitar à análise e tornar mais didática à apresentação. Estes quatro volumes, compreendendo tanques, geradores de gás, bombas e turbinas e a câmara de combustão, foram utilizados para analizar as condições de entrada e saída das propriedades dos fluidos, no caso os propelentes e gases. Como os volumes de controle estão em sequência, as propriedades de saída de um dos volumes analizados são os dados de entarda do próximo.

A análise com base na primeira lei da termodinâmica (princípio de conservação de energia), juntamente com algumas considerações iniciais, culmina ao encontrar a relação entre as vazões mássicas e das entalpias dos propelentes (LOX/LH2) em um motor foguete.

Após este processo, pode-se utilizar a equação para trabalhar com motores reais, encontrando uma vazão mássica a partir de um empuxo predeterminado e utilizando-a para encontrar as outras propriedades termodinâmicas ao longo do motor. Além disso, pode-se aplicar os valores encontrados num software de simulação de motores de foguete, como o Rocket Propulsion Analisys (RPA) ou o Chemical Equilibrium with Applications (CEA), para prever seus parâmetros de desempenho, como velocidade característica, coeficiente de empuxo, entre outros.

\section{Lista de Símbolos}

$\Delta U$ - variação total da energia

$\% m_{L H 2}$ e $\% m_{L O X}$ - fração de massa dos fluidos

$\dot{Q}$ - taxa de transferência de calor

$\dot{W}$ - taxa de transferência de energia por trabalho

$\dot{M}$ - fluxo de massa

$h_{i}$ - entalpia

$V_{i}$ - velocidade

$g$ - aceleração da gravidade

$z_{i}$ - posição vertical

$\eta$ - eficiência

$P_{i}$ - pressão

$I_{s p}$ - impulso específico

$\rho$ - densidade

$H$ - altura de carga em metros

indices

$1,2,3 \ldots-$ pontos na linha de alimentação

amb - ambiente

t- total

\section{Referências}

[1] M. Normah, K.M. Yusof e Z. Tasir, Procedia - Social and Behavioral Sciences 56, 703 (2012).

[2] W. Dempster, C.K. Lee e J.T. Boyle, em Proceedings of the 2002 American Society for Engineering Education Annual Conference 85 Exposition (Washington, 2002).

[3] H.A. Aglan e S.F. Ali, Journal of Engineering Education 85, 327 (1996).

[4] C. Bacha, A. Pellegrino, R. Battista e E. Toson, em 67th International Astronautical Congress (Guadalajara, 2016).

[5] A.T.C. Trevelin, M.A.A. Pereira e J.D. Oliveira Neto, Revista de Estilos de Aprendizaje 6, 12013.

[6] K.B. Blair, D.W. Miller, D.L. Darmofal, P.W. Young e D.R. Brodeur, em Proceedings of the 2002 American 
Society for Engineering Education Annual Conference $\mathcal{E}$ Exposition (Washington, 2002).

[7] M. Srinivasan, M. Wilkes, F. Stevenson, T. Nguyen e S. Slavin, Academic Medicine 82, 74 (2007).

[8] S.L. Queiroz e P.F.O. Cabral, Estudos de caso no ensino de ciências naturais (Centro de Divulgação Científica e Cultural, São Carlos, 2016).

[9] F. Bassoli, Ciência \& Educaçao (Bauru) 20, 579 (2014).

[10] J.C. Passos, Rev. Bras. Ens. Fís. 31, 3603.1 (2009).

[11] M.L.L. Farias e M.A.A. Barbosa, Rev. Bras. Ens. Fís. 39, e4402.1 (2017)

[12] M.V.S. Fonseca, I M.L. Rodrigues e M.B.S. Fonseca, Rev. Bras. Ens. Fís. 40, e3504.1 (2018).

[13] C.F. Wehmann, P.A.C. Rocha, M.E.V. Silva, F.A.A. Araújo e D.L. Correia, Rev. Bras. Ens. Fís. 40, e2313.1 (2018).

[14] L. Goovaerts, M. Cock, K. Struyven, W. Dehaene, European Journal of STEM Education 4, 2 (2018).

[15] M.J.L. Turner, Rocket and spacecraft propulsion: principles, practice and new developments (Springer-Verlag, Berlin, 2009), $3^{\text {a }}$ ed.

[16] G.P. Sutton e O. Biblarz, Rocket propulsion elements (John Wiley \& Sons, New Jersey, 2010), $7^{\text {a }}$ ed.

[17] G. Viggiano, Chandrayaan-2: conheça a missão espacial da Índia para chegar à Lua, disponível em: https://revistagalileu.globo.com/Ciencia/ noticia/2019/07/chandrayaan-2-conheca-missaoespacial-da-india-para-chegar-lua.html, acessado em 01/05/2020.

[18] G. Dvorsky, Missão israelense para a Lua é um marco para o país e para a indústria espacial privada, disponível em: https://gizmodo.uol.com.br/israel-beresheetlua/, acessado em 01/05/2020.

[19] P. Gnipper, Conheça a trajetória das missões Chang'e, que levaram a China à Lua, disponível em: https://canaltech.com.br/espaco/conheca-atrajetoria-das-missoes-change-que-levaram-achina-a-lua-140885/, acessado em 01/05/2020.

[20] O.B. Loureda e J.B.S. Araújo, Revista Latino-Americana de Educação em Astronomia 6, 67 (2008).

[21] M.J. Moran, H.N. Shapiro, D.D. Boettner e M.B. Bayley, Fundamentals of engineering thermodynamics (Wiley, New Jersey, 2010), $7^{\mathrm{a}}$ ed. 